\section{Case Reports in Acute Medicine}

\title{
Tracheal Perforation following Chemotherapy of Diffuse Large B-Cell Lymphoma
}

\author{
Napplika Kongpolprom \\ Division of Pulmonary and Critical Care Medicine, Department of Medicine, Faculty of \\ Medicine, Chulalongkorn University, King Chulalongkorn Memorial Hospital, The Thai Red \\ Cross Society, Bangkok, Thailand
}

\section{Keywords}

Diffuse large B-cell lymphoma · Tracheal perforation · Tracheal disruption

\begin{abstract}
Tracheal perforation is a rare and life-threatening complication following the treatment of lymphoma. There are only a few published cases of tracheal disruption from invasive lymphoma, and most of the patients did not survive. Tumor erosion and nodal necrosis from rapid tumor growth or chemotherapeutic response can cause tracheal disruption. We report here a case of tracheal perforation after chemotherapy of lymphoma. A 69-year-old Thai man presented with upper airway obstruction from a large neck mass encasing and invading the trachea. The patient was intubated to bypass the tracheal obstruction. Biopsy of the mass showed diffuse large B-cell lymphoma. Chemotherapy was urgently administered. Two days after treatment, the patient developed acute respiratory distress with high airway resistance. Emergency bronchoscopy and chest computerized tomography revealed disrupted anterior wall of trachea and endotracheal tube displacement, which created a false track into the mass. Tumor necrosis was thought to be the cause of this perforation and tube malposition. The tube was repositioned by bronchoscopic guidance. Unfortunately, the disrupted trachea could not be reconstructed. Palliative care was consequently provided, and the patient finally passed away a week after the catastrophic event. This report demonstrates clinical presentation, chest imaging, and bronchoscopic findings of tracheal perforation, which is a rare complication following the treatment of lymphoma.




\section{Background}

Tracheal perforation is a rare and life-threatening complication following the treatment of lymphoma. There are only a few published cases [1-4] of tracheal disruption from invasive lymphoma, and most of the patients did not survive. Tumor erosion and nodal necrosis from rapid tumor growth or chemotherapeutic response can cause tracheal disruption. This report demonstrates a lymphoma patient with a large neck mass who initially had upper airway obstruction from tumor invasion, and subsequently had tracheal perforation from tumor necrosis.

\section{Case Presentation}

A 69-year-old Thai man was referred from a primary hospital with upper airway obstruction from a large neck mass. Chest radiography and computerized tomography (CT) showed an anterior neck mass extended to the mediastinum and invaded the trachea and the adjacent organs. The tumor infiltrated along the trachea, from the vocal cords to the carina, narrowing the tracheal lumen, with the narrowest diameter of $0.2 \mathrm{~cm}$. Consequently, the patient was intubated with a 5.5-mm-diameter non-cuffed endotracheal tube (ETT) to bypass the obstruction and assist ventilation. Biopsy of the mass was urgently done and showed diffuse large Bcell lymphoma. Intravenous dexamethasone followed by vincristine was administered before switching to R-CHOP regimen to prevent tumor lysis syndrome. Two days later, the patient developed severe ventilator asynchrony, auto-triggering and delaying ventilator cycling, due to a large amount of air leakage around the ETT. Increased tracheal diameter from chemotherapeutic response was considered; thus, the patient's ETT was replaced with a larger ETT (7.0-mm-diameter tube). The patient was well ventilated after this change for $9 \mathrm{~h}$, whereupon the patient suddenly developed respiratory distress with oxygen desaturation. The ventilation became difficult, despite providing manual ventilation. The ETT was suspected to be obstructed, so the ETT was replaced. However, ventilation was not improved and airway resistance was obviously high. Emergency bronchoscopy revealed granulation-like tissue obstructing the tip of the ETT (Fig. 1). Chest radiography revealed the tip of the ETT was far beyond the lateral wall of the trachea. Chest CT demonstrated tracheal disruption and ETT malposition (Fig. 2). The tube had anteriorly penetrated the perforated site, $6 \mathrm{~cm}$ above the carina, through a false track into the mass. As a result of this life-threatening event, tumor necrosis was suspected to be the primary cause of tracheal perforation and it was considered that tube reexchanging might have further created the new track into the tumor. Consequently, repositioning of the ETT guided by bronchoscopy was performed and the tube was successfully placed to bypass the perforated site. Unfortunately, the disrupted trachea could not be reconstructed due to the necrotic tumor around the lesion. Additionally, in view of the rapid change of tracheal diameter, tracheal stent was not inserted to avoid stent migration. Finally, the patient was ventilated via the ETT under deep sedation. The aim of treatment was then changed to palliative care due to the aggressive nature of the tumor and the untreatable complication. The patient subsequently passed away a week after this catastrophic perforation. 


\section{Discussion and Conclusions}

For more than 20 years, tracheal perforation has been reported in patients with nodal lymphoma and thyroid lymphoma [1-4]. Diffuse large B-cell lymphoma is the most common subtype of lymphoma involving the trachea [5]. Tracheal disruption may result from the lymphoma itself or its treatment. In contrast to lymphoma involving the gastrointestinal tract [6], perforation of the tracheobronchial tree is rare and the prognosis of patients with tracheal perforation is very poor. Almost all of the reported patients died as a result of this complication. There is only one report of a patient with tracheal perforation who survived due to spontaneous fibrotic healing [1].

This patient's clinical presentation and the aggressive behavior of the tumor suggested anaplastic thyroid cancer as the most likely initial diagnosis. However, lymphoma is not uncommon and cannot be differentiated from thyroid cancer merely by clinical manifestation; thus, it was not ruled out [7].

Patients with thyroid lymphoma, nodal lymphoma, and mucosa-associated lymphoid tissue (MALT) may present with a neck mass involving the trachea. Upper airway obstruction appears in some patients. It results from either mass compression, vocal cord paralysis due to tumor involving the recurrent laryngeal nerve, or tracheal stenosis due to tumor infiltration.

The incidence of thyroid lymphoma is less than $5 \%$ of thyroid cancer and $1-2 \%$ of extranodal lymphomas [5]. Additionally, thyroid lymphoma is associated with a history of Hashimoto's thyroiditis and causes diffuse thyroid enlargement with or without the extension into adjacent organs. Moreover, necrosis and extensive invasion are atypical features of thyroid lymphoma.

In contrast, nodal lymphoma originates outside the thyroid gland and can invade the thyroid and contiguous organs. Aggressive invasion and necrosis is more common in nodal lymphomas, while MALT arises in the thyroid gland or trachea and usually presents with localized disease.

For a definite diagnosis, pathological examination and immunohistochemistry are essential. However, there are useful clues in CT findings that can help differentiate types of the primary tumor [8]. Thyroid cancer and thyroid lymphoma both present with thyroid enlargement or thyroid mass. However, lymphoma appears homogeneous, whereas thyroid cancer usually has heterogeneous attenuation, necrosis, and calcification [9]. For this reported patient, chest CT demonstrated homogeneous tumor originating outside the thyroid gland, displacing the thyroid gland laterally. These findings were compatible with nodal lymphoma.

With regards to the course of treatment, the reported patient encountered two critical airway problems, namely tracheal stenosis and tracheal perforation. Although a tracheal stent would have been the best option for maintaining airway patency [10-13] and occluding tracheal fistula [14], there were several limitations for applying a stent in this patient. Firstly, the length of the tracheal lesion would have led to incomplete maintenance of the airway caliber. Secondly, the lumen was too narrow for any instrument, especially a rigid bronchoscope, to be inserted. Lastly, the tumor infiltrated along the trachea, close to the vocal cords; thus, inserting a stent to cover the lesion in this area would have blunted vocal cord reflexes. Taking all factors into account, a small-sized ETT was inserted instead of a stent.

At the time of sealing the perforated trachea, high risk of stent migration was a concern owing to the irregular tracheal size. Therefore, bypassing the defect by ETT was the most viable option to manage the disrupted trachea.

In this case study, there were multiple limitations for airway manipulation. Furthermore, the patient may not have been able to tolerate the complications from a full chemotherapeutic 
regimen. Following the multidisciplinary team of physicians' discussion with the patient's family, it was decided that only palliative care would be provided to the patient.

In conclusion, tracheal perforation is a rare complication of lymphoma. Physicians should be vigilant of airway disruption in patients with lymphoma infiltrating or eroding the tracheobronchial tree, even in those responding well to treatment. Prognosis of patients with such complications remains very poor despite early diagnosis due to limitations of current available treatments.

\section{Statement of Ethics}

The case presentation was approved by the Institutional Review Board, Faculty of Medicine, Chulalongkorn University (IRB No. 706/60). Written informed consent was obtained from the next of kin for publication of this case report. A copy of the written consent is available for review by the Editor-in-Chief of this journal.

\section{Disclosure Statement}

The author declares that she has no competing interests.

\section{References}

1 Chen C, Tibbetts KM, Tassler AB, Schiff BA. Tracheal invasion and perforation from advanced primary thyroid lymphoma: a case report and literature review. Am J Otolaryngol. 2013 Sep-Oct;34(5):559-62.

2 Hugh GA, Christopher R. Morse. Tracheal disruption from invasive pretracheal lymphadenopathy. Ann Thorac Surg. 2013;95 e23.

3 Jaremko JL, Rawat B, Naik S. Oesophageal and tracheal perforation in thyroid B-cell lymphoma. Australas Radiol. 2007 Dec;51 Suppl:B193-5.

4 Melnyk A, Graham NJ, Weber D, Walsh GL. Lethal tracheal dissolution during treatment for thyroid lymphoma. Thorax. 1995 Oct;50(10):1120-1.

5 Vega F, Lin P, Medeiros LJ. Extranodal lymphomas of the head and neck. Ann Diagn Pathol. 2005 Dec;9(6):340-50.

6 Vaidya R, Habermann TM, Donohue JH, Ristow KM, Maurer MJ, Macon WR et al. Bowel perforation in intestinal lymphoma: incidence and clinical features. Ann Oncol. 2013 Sep;24(9):2439-43.

7 Kennedy KS, Wilson JF. Malignant thyroid lymphoma presenting as acute airway obstruction. Ear Nose Throat J. 1992 Aug;71(8):350, 353-5.

8 Ishikawa H, Tamaki Y, Takahashi M, Higuchi K, Sakaino K, Nonaka T et al. Comparison of primary thyroid lymphoma with anaplastic thyroid carcinoma on computed tomographic imaging. Radiat Med. 2002 JanFeb;20(1):9-15.

9 Lee JW, Yoon DY, Choi CS, Chang SK, Yun EJ, Seo YL et al. Anaplastic thyroid carcinoma: computed tomographic differentiation from other thyroid masses. Acta Radiol. 2008 Apr;49(3):321-7.

10 Ding J, Chen Z, Shi M. Tracheal stenting for primary tracheal mucosa-associated lymphoid tissue lymphoma. Eur J Med Res. 2013 Apr;18(1):8.

11 Lee J, Won JH, Kim HC, Soh EY. Emergency dilation by self-expandable tracheal stent for upper airway obstruction in a patient with a giant primary thyroid lymphoma. Thyroid. 2009 Feb;19(2):193-5.

12 Myatt HM. Acute airway obstruction due to primary thyroid lymphoma. Rev Laryngol Otol Rhinol (Bord). 1996;117(3):237-9.

13 Oki M, Saka H. Temporary use of silicone stents for severe airway stenosis in untreated malignant lymphoma. J Bronchology Interv Pulmonol. 2013 Jan;20(1):21-7.

14 Choudhary C, Gildea TR, Salman R, Guzman ED, Mehta AC. Management of tracheomediastinal fistula using self-expanding metallic stents. Ann Thorac Surg. 2008 May;85(5):1800-2. 


\section{Case Reports in Acute Medicine}

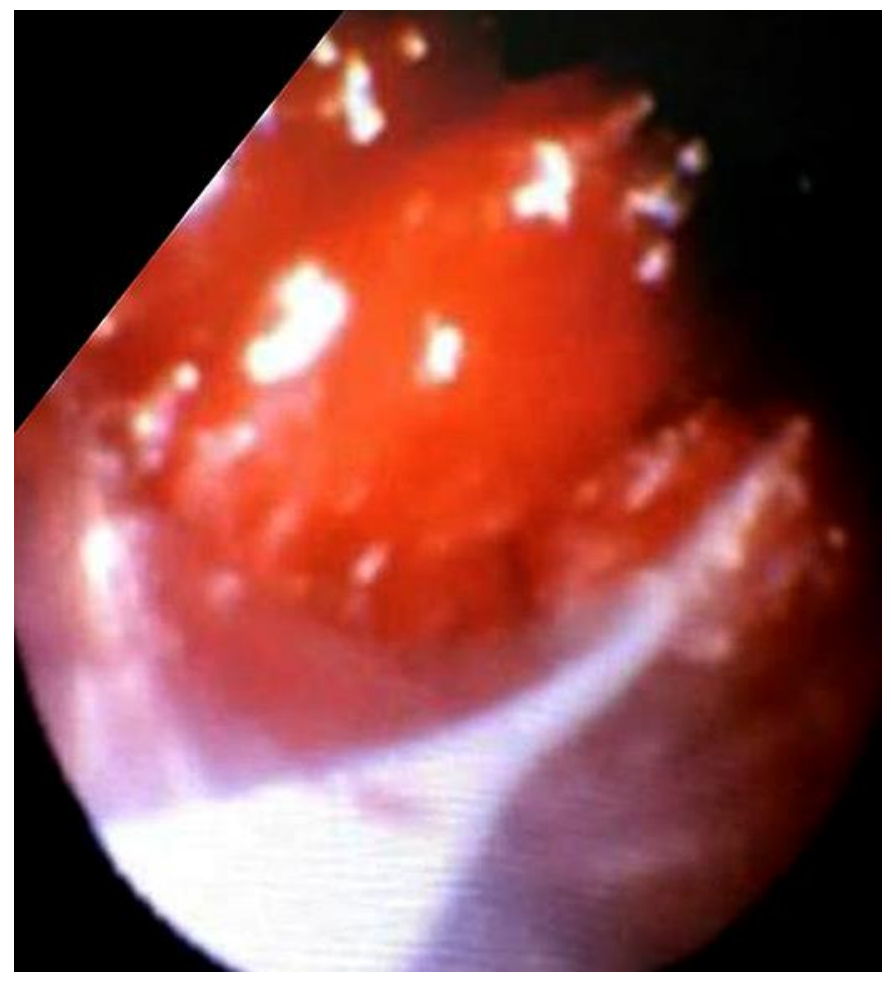

Fig. 1. Bronchoscopic findings during the repositioning of the ETT. Bronchoscopic findings reveal granulation-like tissue at the tip of the ETT and the false track distal to this tissue.

Kongpolprom: Tracheal Perforation following Chemotherapy of Diffuse Large B-Cell Lymphoma 

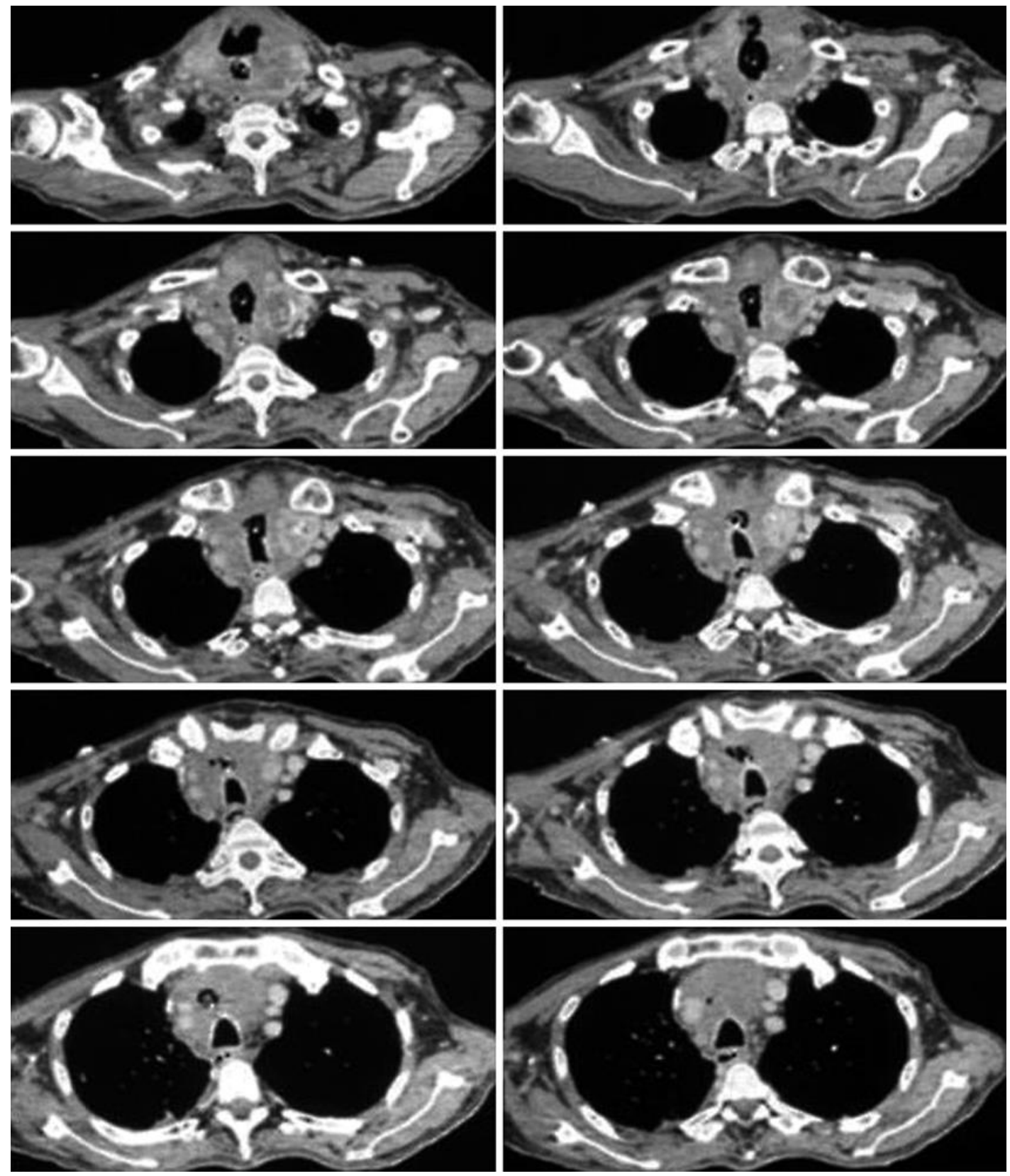

Fig. 2. Chest CT at the critical event. Chest CT reveals malposition of the ETT. The ETT anteriorly penetrates the disrupted tracheal wall to the mass in the superior mediastinum. 